\title{
Seeing Big with Scanning Electrochemical Microscopy
}

\section{Specialized microelectrode probes fabricated in a soft polymer film now make it possible to use scanning electrochemical microscopy to image the reactivity of large, corrugated, tilted, and dry surfaces. (To listen to a podcast about this Feature, please go to the Analytical Chemistry multimedia page at pubs.acs.org/page/ancham/audio/index.html.)}

\author{
Fernando Cortés-Salazar, Dmitry Momotenko, and Hubert H. Girault \\ Ecole Polytechnique Fédérale de Lausanne (Switzerland)
}

\section{Andreas Lesch and Gunther Wittstock}

Carl von Ossietzky University of Oldenburg (Germany)

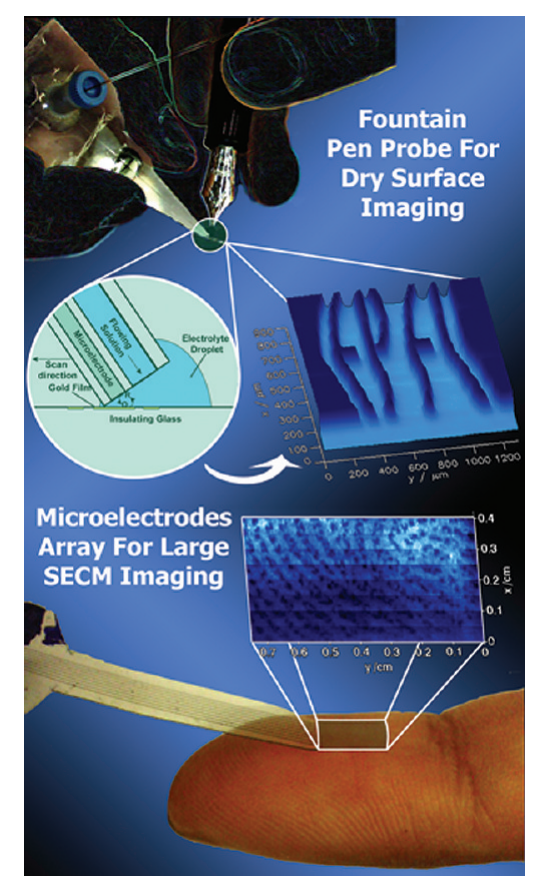

canning electrochemical microscopy (SECM) has evolved as $\checkmark$ a versatile tool for the spatial characterization of surface reactivity and mass fluxes at solid-liquid, liquid-liquid, and liquid-gas interfaces. ${ }^{1-3}$ Its applications include imaging patterned sensor surfaces, ${ }^{4}$ measuring substance uptake and release from biological cells, ${ }^{5}$ investigating very fast electrochemical reactions, ${ }^{6}$ studying local corrosion, ${ }^{7}$ screening electrocatalysts, ${ }^{8}$ analyzing forensic samples, ${ }^{9}$ and modifying surfaces. ${ }^{10}$ The most common probes are amperometric microelectrodes produced by sealing a Pt wire of 10 or $25 \mu \mathrm{m}$ diameter in a glass capillary and exposing the cross-section by grinding and polishing. The microelectrode is attached to a positioning system that provides translation of the probe in horizontal $(x, y)$ and vertical $(z)$ directions. During scanning, a steady-state faradaic current is measured with a (bi)potentiostat. The faradaic reaction results from the electrolysis of diffusing redox-active species undergoing electrochemical reactions at both the microelectrode and the local reaction sites on the specimen surface. The measured currents reflect the interplay between mass transport by diffusion and heterogeneous reactions at the sample. For many situations, they can be calculated by continuum simulation. ${ }^{11-13}$ Quantitative kinetic information can be extracted from comparison of experimental data with such simulation.

Typical optical microscopy and other scanning probe techniques such as atomic force microscopy (AFM) or scanning tunneling microscopy (STM) differ from SECM by the physical principles of operation and thus by the type of information extracted. ${ }^{14}$ Additionally, SECM can be applied to study samples that are challenging for optical microscopy such as multicolored surfaces and porous materials. The major advantage of SECM is its ability to probe local surface reactivity with lateral resolution and high sensitivity. ${ }^{15}$ Indeed, SECM provides researchers in many fields with an important tool for mapping chemical and electrochemical processes occurring at almost any interface. ${ }^{16,12}$

However, the steady-state current recorded during scanning depends on the probe-substrate distance, $d$. Therefore, corrugated, tilted, and curved samples yield experimental artifacts and are difficult to image. In addition, when closely scanning over such surfaces, probe-substrate crashes can destroy the commonly used glass microelectrodes. This may require additional equipment for constant distance images or special samples and alignment procedures to adjust the sample plane parallel to the $x, y$-plane of the positioning system. Moreover, when the sample area is larger than a few square millimeters, the recording time for such a large image frame is extremely long, i.e., $>10 \mathrm{~h}$. Consequently, solvent evaporation, electrode fouling, and sample aging limit the use of SECM. Although SECM imaging of samples covered only by a thin film of condensed moisture has been reported, ${ }^{17,18}$ the typical SECM experiment aims to exploit the redox chemistry of samplegenerated or deliberately-added redox systems, which requires the immersion of the sample surface into an electrolyte solution. The latter procedure becomes cumbersome for large, bent, or delicate sample surfaces that need to be investigated and from which smaller samples cannot be taken: for example, testing coated metal pieces of working machines at regular intervals.

Published: February 07, 2011 
This feature describes new SECM probes developed to overcome these limitations that allow the analysis of large image frames and/or samples of complicated topology without loss of resolution compared to conventional glass-shielded microelectrodes. ${ }^{19-21}$ The new probes are made by classical microfabrication techniques and are appropriate for a wide range of samples, thereby broadening the applicability of SECM to new areas.

\section{SOFT STYLUS PROBES TO IMAGE TILTED AND CORRUGATED SURFACES}

The response of a SECM probe strongly depends on the surface reactivity of the sample and the distance, $d$, between the surface and the active area of the probe electrode. To obtain a reactivity image that is not influenced by topography, $d$ must be constant. In conventional SECM operations, this can be achieved by analyzing samples with a roughness that is considerably smaller than the radius of the microelectrode, $r_{\mathrm{T}}$, and by leveling the plane of the sample surface with respect to the $x, y$-scanning plane of the positioning system. However, this approach becomes inappropriate on rough surfaces or when investigating large image frames (in the square centimeter range); similar limitations apply when imaging curved samples.

In the case of a small $r_{\mathrm{T}}$, a constant $d$ can be maintained by coupling SECM with scanning ion conductance microscopy (SICM), ${ }^{22}$ by working in a tip-position modulation SECM mode with a supplementary system that allows close feedback loop positioning of the probe, ${ }^{23,24}$ or by combining SECM with AFM (AFM-SECM $)^{25-27}$ or electrochemical tunneling microscopy (EC-STM). ${ }^{28}$ The integrated AFM-SECM probes proposed by Kranz et al. contain an insulating thorn that is used for AFM imaging and at the same time defines a constant $d$ for the frame electrode exposed by a focused ion beam. ${ }^{29}$ Metal nanowire AFM-SECM probes have been built by coating single wall carbon nanotubes with metal, insulating them with a polymer film, and exposing the final electrode surface with a focused ion beam. ${ }^{30}$ Tilting the disk-shaped probe establishes a physical contact between one side of the insulating probe apex and the substrate while the active electrode area remains at a given distance from the sample. These nanowire AFM-SECM probes have been used in intermittent contact mode for high resolution imaging of both topographical and electrochemical surface information. ${ }^{30}$

For rough surfaces and for sample areas larger than the typical scanning range of AFM instruments (e.g., $100 \mu \mathrm{m} \times 100 \mu \mathrm{m} \times$ $10 \mu \mathrm{m})$, a distance control has been achieved by shear-force detection in combination with a positional feedback system. ${ }^{31,32}$ Since the shear forces must be measured in a viscous liquid, the signal change upon approach is rather small. Therefore, long integration times restrict the lateral translation rate, which becomes limiting when imaging large areas. Alternating current SECM (AC-SECM) has also been proposed to circumvent these difficulties. However, the distance measurement is difficult to interpret quantitatively because it depends on the electrochemical cell setup, the nature of the sample, frequency, and electrolyte conductivity. ${ }^{33-35}$

The problems of scanning tilted, rough, or curved samples can be circumvented by using microelectrodes fabricated in soft polymeric materials that allow the probe to scan the sample in a contact regime. Figure 1a illustrates the concept and use of the soft stylus probe for SECM in a contact regime. As shown in Figure 1, when scanning in a contact regime over a substrate, recycling of a redox mediator is only achieved when the bent probe is in the neighborhood of a conductive surface. This clearly
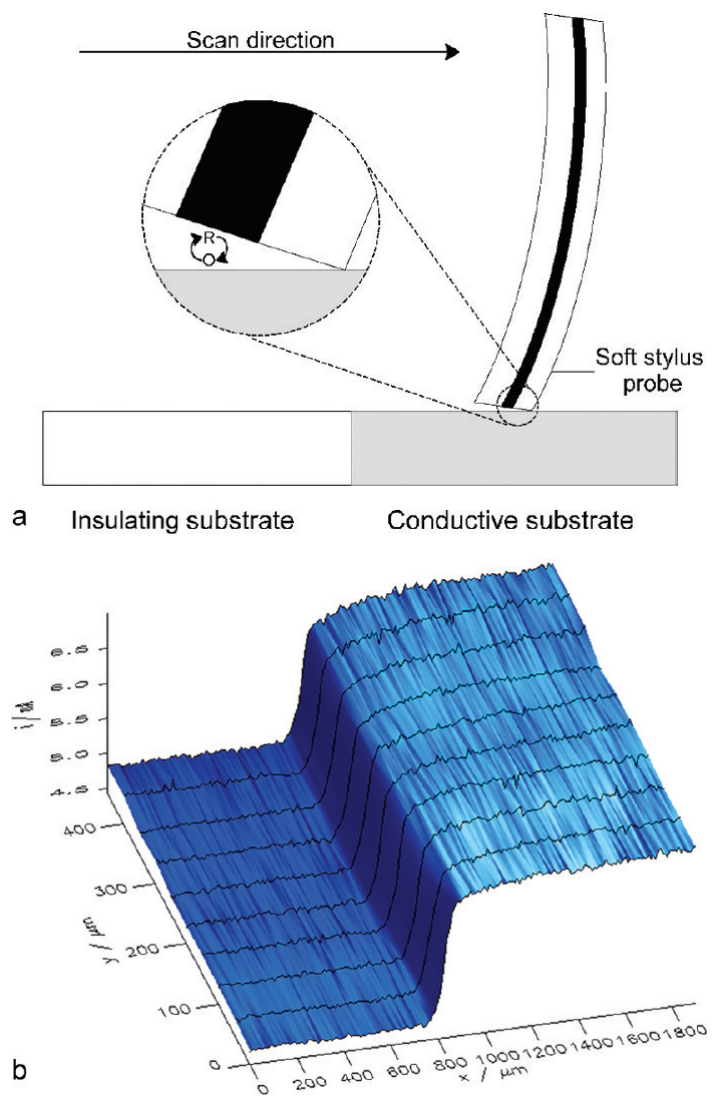

Figure 1. a) SECM principle of surface reactivity characterization with a soft stylus probe working in a contact regime. b) SECM image in a contact regime of a partially gold-covered glass surface obtained by using a soft stylus probe. Imaging conditions: $2.1 \mathrm{mM} \mathrm{FcCH}_{2} \mathrm{OH}, 0.1 \mathrm{M}$ $\mathrm{KNO}_{3}$. Working electrode potential $E_{\mathrm{T}}=0.3 \mathrm{~V}$, step size in $x$ and $y$ directions was 10 and $50 \mu \mathrm{m}$, respectively. Translation rate $v_{\mathrm{T}}=10 \mu \mathrm{m} \mathrm{s}^{-1}$.

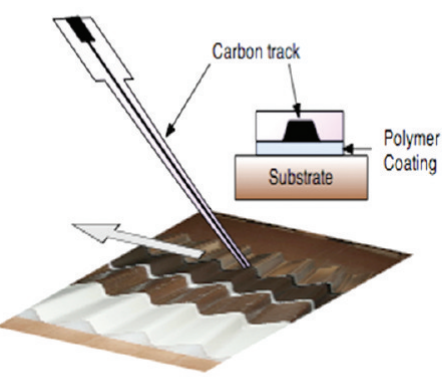

a

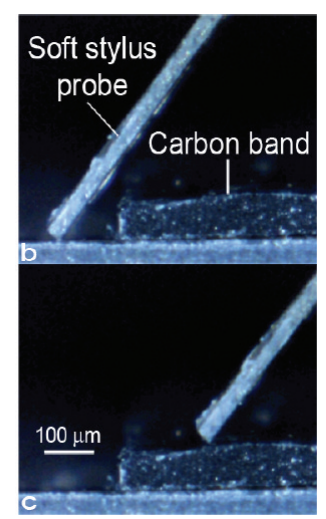

Figure 2. a) Schematic representation of a soft stylus probe used as a working electrode for SECM in a contact regime. b-c) Optical image of the soft stylus probe scanning in a contact regime above an unbiased carbon band with a height of $118 \mu \mathrm{m}$.

illustrates the possibility of surface reactivity imaging with the soft stylus probe approach. The schematic representation of the probe shown in Figure 2a depicts the soft stylus that was microfabricated by ablating a microchannel on a polyethylene terephthalate (PET) sheet and filling it with carbon ink. Lamination 
of the cured carbon track with a polyethylene/PET film completes the probe. To obtain the microelectrode, either laser or blade cutting exposes a cross-section of the carbon track, followed eventually by mechanical polishing. The probe contact angle and the lamination film thickness when scanned in a contact regime define the probe-substrate distance (see Figure 1a, 2b, and 2c). This provides a constant $d$ between the active electrode area and the sample surface without the use of an electronic feedback loop for vertical positioning. ${ }^{19}$ The main advantage of scanning in a contact regime is that a small and almost constant $d$ can be maintained without tip or sample destruction by probe-substrate crashes. In addition, the broad range of soft plastic materials that can be used in microfabrication processes allows the tuning of the probe stiffness to avoid the destruction of even modestly fragile samples.

The probe-substrate distance can be reduced by choosing thin polymer coating materials and by adjusting the contact angle when the probe is pressed against the substrate. A smaller $d$ results in a higher current contrast. Levelling the sample before SECM imaging is not required when working in a contact regime, making the setup of SECM experiments much faster and easier. Additionally, soft stylus probes can be employed in a contactless regime as they are normally used during SECM experiments, showing the versatility of the soft stylus probes in different SECM applications. ${ }^{19}$

Soft stylus probes with different active electrode materials can be easily fabricated by modern microfabrication methods such as photolithography or laser photoablation. Like normal microelectrodes sealed in glass, the soft probes can be used many times. As seen in Figure $2 b-c$, the bent probe can scan over 3D patterns as thick as a $118 \mu \mathrm{m}$ without drastically changing $d$ or destroying the probe, which allows imaging the chemical reactivity of rough surfaces regardless of the topography. ${ }^{19}$

\section{- FOUNTAIN PEN PROBE TO IMAGE DRY SURFACES}

In classical SECM, the sample should be placed within an electrochemical cell filled with electrolyte solutions. This is a major drawback in comparison to other scanning probe methods such as AFM and STM. Furthermore, when imaging over long periods of time, solvent evaporation becomes a problem. Therefore, different approaches have been proposed to circumvent these inherent difficulties. ${ }^{36-38}$

Integrated three-electrode ${ }^{36}$ or two-electrode ${ }^{37,38}$ cells have been introduced to simplify the electrochemical cell design, to prevent collisions between electrodes, and to allow an easier positioning of the electrochemical cell. To avoid experimental artifacts from solvent evaporation (e.g., change in solute concentration and precipitation processes), different approaches have been proposed. ${ }^{36-38}$ Lohrengel et al. have built a three-electrode setup in which the studied surface acts as a working electrode while reference and counter electrodes are contained in a capillary that delivers an electrolyte droplet. ${ }^{36}$ In this case, the area covered by the electrolyte droplet defines the active electrode area of the working electrode, which at the same time limits this device to the study of conductive surfaces. Spaine et al. introduced a positionable microcell, in which the reference and working electrodes were localized separately in one of the channels of a theta glass capillary (a capillary tubing that is divided into two hemicylindrical sides by a central glass septum).$^{37} \mathrm{~A}$ carbon fiber sealed with epoxy was the working electrode, while a $\mathrm{Ag} / \mathrm{AgCl}$ wire was positioned as the reference electrode in the other compartment with a concentrated solution of $\mathrm{NaCl}$ and a salt bridge at the bottom of the glass capillary. Although voltammetry and SECM measurements were

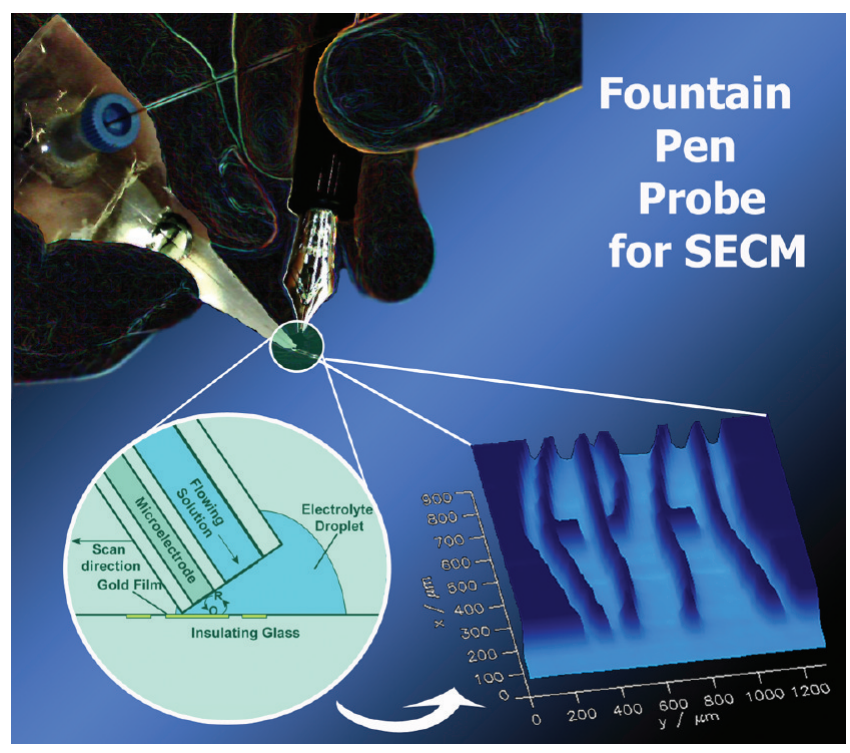

Figure 3. Optical image of a fountain pen that enables SECM imaging of dry surfaces by delivering microliter volumes of mediator solution into a miniaturized two-electrode cell (upper left). Principle of surface reactivity characterization with a fountain pen (lower left). SECM image of a gold micro EPFL (Ecole Polytechnique Fédérale de Lausanne) logo taken with a polished fountain pen in feedback mode (lower right).

performed successfully at subnanoliter volumes, the configuration was cumbersome, and high expertise was required.

Recently, Turcu et al. have reported a simple way of preparing a two-electrode cell by coating a common glass-encapsulated microelectrode with a Ag film. ${ }^{38}$ Thus, successful SECM imaging was carried out in a nanoliter droplet of mediator solution covered with a layer of paraffin oil to avoid solvent evaporation problems. The paraffin film is a potential source of electrode contamination and restricts the scanned area because physical barriers are needed to avoid paraffin or redox mediator leakage. Working in a watersaturated atmosphere within a humidity chamber or adding glycerin to the supporting electrolyte can also slow solvent evaporation. ${ }^{39,37}$

Junker et al. applied a multipurpose microfluidic probe concept to a microfluidic system in which two open channels are employed for local delivery and aspiration of a solution over the sample surface. ${ }^{40}$ Fluidic channels also have been introduced into the AFM cantilever, allowing fluid transport from a reservoir to the end of the cantilever. ${ }^{41-44}$ Leïchle et al. used an electrically contacted cantilever with integrated microfluidics for localized copper deposition. ${ }^{45}$

More recently, a fountain pen probe for SECM that integrates a microfluidic system with a two-electrode cell has been proposed. ${ }^{20}$ The fountain pen is made by the same microfabrication technology as the soft stylus probes described above ${ }^{19}$ but introduces a microfluidic channel and a counter/reference electrode prepared by ablating a parallel and aligned second microchannel on the opposite side of the carbon track used as working electrode. For surface reactivity characterization, the fountain pen is used in the contact regime similar to the soft stylus probe. Then microliter volumes of mediator solution are delivered into the miniaturized two-electrode cell—-like a fountain pen—allowing the surface reactivity characterization of dry surfaces (Figure 3, left). When the probe is biased to a potential in which the redox mediator electrolysis is performed under diffusion-controlled conditions and is scanned over an insulating substrate, a low current is recorded as a consequence of the hindered diffusion (negative feedback). 
When the probe is scanned over a conductive substrate, recycling of the mediator (positive feedback) causes current to increase. A concentration cell with cathodic and anodic regions on the wetted surface regions (Figure 3, lower left) provides the driving force. ${ }^{46}$

As a proof-of-concept, the fountain pen probe was employed for surface reactivity imaging in the feedback mode of a gold on glass pattern, as shown in the lower right of Figure 3 (EPFL logo). The recording time of this image was $2 \mathrm{~h} 15 \mathrm{~min}$, during which no apparent changes of the current caused by the evaporation process (i.e., drastic increase of the redox mediator concentration) were observed when scanning over the dry surface. Thus, problems in microenvironment SECM studies related to evaporation processes could be counterbalanced by the constant flow of solution from the open microchannel. In addition, the simplified two-electrode cell design circumvents all the constraints for SECM experiments in microvolumes and extends the scope of SECM to the scanning of dry surfaces.

\section{SECM SCANNING WITH MULTIPLEXED PROBES}

Several applications of interest such as protein arrays, ${ }^{47}$ human fingerprints, ${ }^{9}$ electropherogram read-out, ${ }^{48}$ quality control of coatings, ${ }^{7}$ and screening of combinatorial libraries ${ }^{49-51}$ could require image frames in the square centimeter range. The challenges in imaging large area samples stem from the difficulties in handling tilted, corrugated, and curved samples and from electrode fouling, solvent evaporation, and sample aging introduced by the long recording times. To overcome these problems, three different approaches have been proposed.

Translation rates up to $500 \mu \mathrm{m} \mathrm{s}^{-1}$ with step sizes of $150 \mu \mathrm{m}$ have reduced the recording time for large areas but with loss of image resolution and introduction of convective effects. ${ }^{49,52,53} \mathrm{~A}$ second strategy for reduction of evaporation and electrode fouling is to image sub-regions of large samples followed by renewal of supporting electrolyte and electrode surface between image frames. ${ }^{54,49}$ However, overall recording time cannot be reduced in this way. A third strategy is to use multiple probe arrays, which have already been applied to AFM imaging of large areas with a clear decrease of the recording time. ${ }^{55,56}$ A similar approach with SECM used a 2D microelectrode array to image samples in the generation-collection mode. ${ }^{57}$ The problem of diffusional shielding of individual electrodes was solved for a confined etchant layer technique by periodically renewing the solution between probes and sample and limiting the extension of the diffusion layer with a homogeneous scavenger reaction. ${ }^{58}$ Barker et al. reported a multielectrode for parallel SECM imaging that comprised a linear array of 16 individually addressable microelectrodes placed on a planar insulating chip. ${ }^{59} 10 \mu \mathrm{m}$ diameter $\mathrm{Pt}$ microelectrodes were prepared by laying a nanometer-thick Pt layer over an insulating silicon nitride film, followed by the application of a second thin nitride film. After exposure of the slightly recessed contact pads and reference and working electrodes, the microelectrode array was fixed below a positioning system that moved the sample horizontally. SECM imaging was successfully demonstrated with this multiprobe. The use of this approach is limited because renewing the active electrodes by mechanical polishing is difficult. ${ }^{59}$

As shown above, the concept of soft stylus probes provides an interesting approach to image rough surfaces. ${ }^{19}$ By multiplexing the readout of the electrodes with a probe array, the recording time for imaging a large area sample can also be reduced drastically without compromising the image quality, while avoiding the related

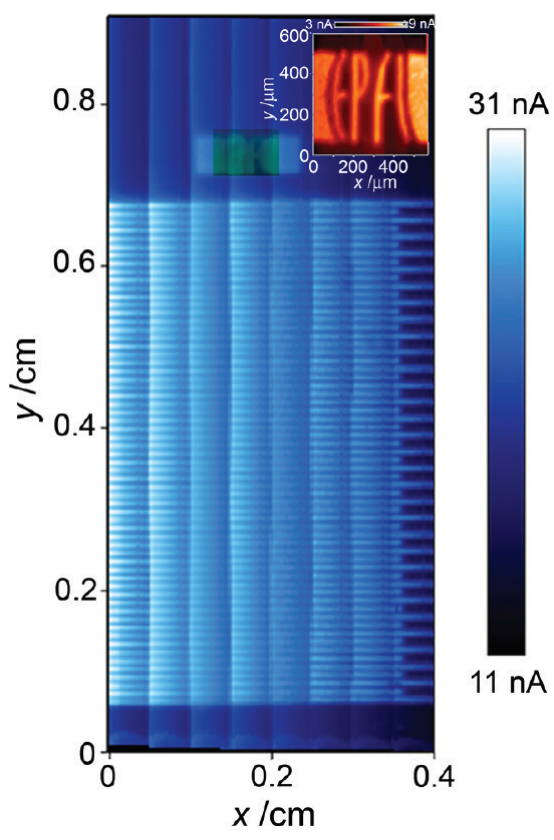

Figure 4. SECM image of a gold printed interdigitated array taken with an array of eight microelectrodes $(500 \mu \mathrm{m}$ electrode-to-electrode spacing) in feedback mode (acquisition time $=5 \mathrm{~h}$ ). Step size $=$ $25 \mu \mathrm{m}$ and translation rate $v_{\mathrm{T}}=25 \mu \mathrm{m} \mathrm{s}^{-1}$ in $2 \mathrm{mM} \mathrm{FcCH}_{2} \mathrm{OH}$ with $0.1 \mathrm{M} \mathrm{KNO}_{3}$. The inset is an enlargement of the highlighted area (green) with a multiprobe array of eight electrodes (130 $\mu \mathrm{m}$ electrodeto-electrode spacing) in feedback mode (acquisition time $=40 \mathrm{~min}$ ). $E_{\mathrm{T}}=0.35 \mathrm{~V}, h_{\mathrm{P}}=-50 \mu \mathrm{m}$, step size $=10 \mu \mathrm{m}$, and translation rate $v_{\mathrm{T}}=$ $10 \mu \mathrm{m} \mathrm{s}^{-1}$ in $2.0 \mathrm{mM} \mathrm{FcCH}_{2} \mathrm{OH}$ with $0.1 \mathrm{M} \mathrm{KNO}_{3}$.

experimental problems outlined above. Thus, the same microfabrication methods employed for fabricating soft stylus probes have produced linear arrays of microelectrodes. ${ }^{21}$ As a consequence, all the advantages provided by performing SECM in a contact regime are combined with a multiplexed probe array.

An important issue when working with arrays is the cross-talk between electrodes, which can be avoided if the thickness of the diffusion layer of each microelectrode is smaller than half the distance between adjacent electrodes. ${ }^{60}$ With this aim, Barker et al. employed an interelectrode spacing equal to $120 \mu \mathrm{m}$ for a $10 \mu \mathrm{m}$ diameter microelectrode, which should be sufficient for avoiding diffusion layer overlapping. However, lower than expected currents were observed by cyclic voltammetry, which could indicate an overlap of the diffusion layers of neighboring electrodes. The latter is most likely due to the fact that the diffusional independence of microelectrodes in arrays is only valid for a finite time scale determined by the distance between neighboring probes. ${ }^{61,62,60}$ Because soft microelectrode linear arrays are obtained as the cross-section of a planar structure rather than on the surface of a chip, the problems of diffusional shielding are reduced and contact with the linear array elements is greatly simplified. Finite element method simulations have predicted that even soft microelectrode arrays presenting quartermoon shape electrodes (i.e., $40 \mu \mathrm{m}$ width and $20 \mu \mathrm{m}$ depth) with $500 \mu \mathrm{m}$ electrode-to-electrode distance will develop an overlapping of the diffusion layers. ${ }^{21}$ However, for such interelectrode spacing, insignificant current differences are recorded at the inner and outer electrodes, allowing qualitative SECM imaging.

Linear soft microelectrode arrays are used by "combing" the surface of the sample. Figure 4 shows a SECM image of an 


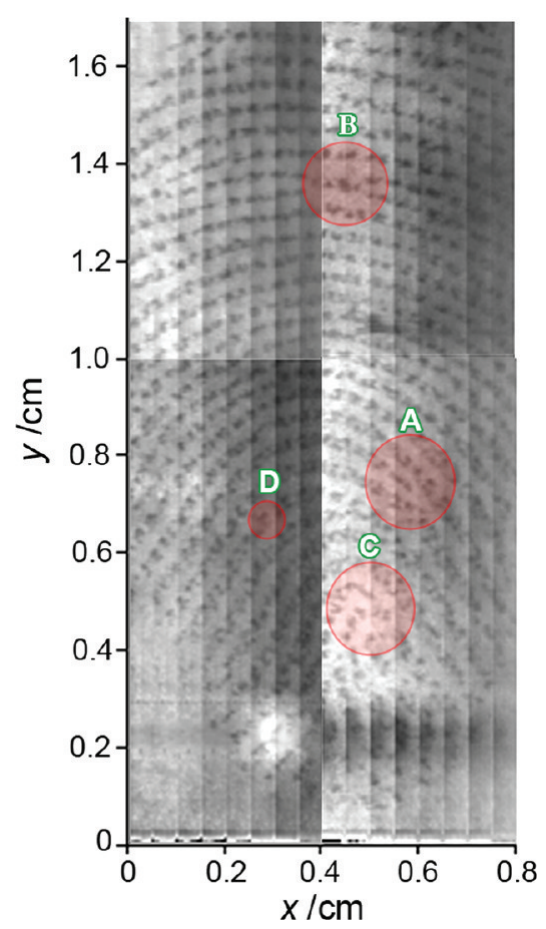

Figure 5. SECM feedback image of a human fingerprint developed by benzoquinone tagging with an array of eight microelectrodes of $500 \mu \mathrm{m}$ interelectrode spacing. $E_{\mathrm{T}}=-0.35 \mathrm{~V}$, step size $=50 \mu \mathrm{m}$, and translation rate $v_{\mathrm{T}}=50 \mu \mathrm{m} \mathrm{s}^{-1}$ (acquisition time $=5 \mathrm{~h} 12 \mathrm{~min}$ ) in $2 \mathrm{mM}$ $\mathrm{K}_{3}\left[\mathrm{Fe}(\mathrm{CN})_{6}\right]$ with $0.1 \mathrm{M} \mathrm{KCl}$.

interdigitated array of gold microelectrodes scanned by a linear array of eight carbon microelectrodes ( $500 \mu \mathrm{m}$ electrode spacing) that was obtained in only $5 \mathrm{~h}$. The recording time is directly proportional to the number of multiplexed electrodes, and therefore 256 electrodes should reduce the time to $10 \mathrm{~min}$. The gold band width and spacing between gold patterns is equal to $50 \mu \mathrm{m}$, which represents a very high resolution when taking into account the dimension of the complete area scanned $(0.9 \times 0.4 \mathrm{~cm})$. At the top of Figure 4, a gold on glass EPFL logo (green) can be identified but is not perfectly resolved because of its more complex structure and smaller size. To obtain a clearer image of this zone, a SECM image in contact mode was acquired by using the array of eight microelectrodes with an electrode-toelectrode distance of $130 \mu \mathrm{m}$ (Figure 4, inset). Despite the diffusion layer overlapping among neighboring microelectrodes in this microelectrode array, high quality SECM images can be attained.

To illustrate further the potential of a linear array of soft microelectrodes, Figure 5 shows the SECM image of a complete human fingerprint. The image is based on the detection of a protein-inked fingermark deposited on a PVDF membrane and tagged with benzoquinone. ${ }^{63}$ The microelectrodes carried out the diffusion-controlled reduction of ferricyanide. At the sample areas tagged with benzoquinone, benzoquinone molecules are reduced and the oxidized mediator is regenerated. ${ }^{63}$ Positive feedback is encountered when scanning protein-containing regions of the fingerprint, while negative feedback is developed for regions without adsorbed proteins.

Human fingerprints are valuable tools for personal identity verification because each individual fingerprint has unique features such as the flow of the ridges (i.e., the overall pattern), the ridge path deviations (e.g., ridge endings and bifurcations marked in Figure 5 as zones $A$ and B, respectively), and the intrinsic ridge characteristics (e.g., ridge shape and pores marked in Figure 5 as zones $\mathrm{C}$ and $\mathrm{D}$, respectively). Recently it has been shown that SECM provides forensic scientists a new tool for the visualization of human fingerprints on unusual surfaces (e.g., multicolored backgrounds, those contaminated by body fluids or other components, and porous surfaces) with high resolution and sensitivity. ${ }^{64,65,9}$ However, approximately $1-2$ days would be required to image a full fingerprint with a single electrode. This excessively long time restricts the use of SECM for forensic purposes. The fingerprint shown in Figure 5 was constructed from four different images taken in a total experimental time of 5 h 12 min, showing the feasibility of using SECM for such a task.

\section{SEEING THE BIG HORIZON WITH SECM}

SECM is increasingly becoming a popular technique to study chemical surface reactivity thanks to its well-developed theory and recent instrumental advances. SECM has been positioned among other scanning probe techniques as an important tool for studying biological samples, screening combinatorial libraries of catalysts, and analyzing protein arrays. Additionally, SECM can be successfully applied to samples ranging from nano to macro dimensions.

Scanning large sample areas is now becoming possible with the use of soft microelectrode arrays as SECM probes. Amperometry is a technique that can easily be multiplexed, so hundreds or even thousands of microelectrodes could be used for imaging. Electrochemical scanners based on this concept will undoubtedly provide new tools for surface analysis and surface patterning in biological and nano- and microtechnology sciences. The application of the soft stylus probe concept should increase the use of SECM because the method is simple and specialized microelectrodes could be prepared easily by modifying the carbon ink with electrocatalysts such as Pt nanoparticles.

Combination of microfluidic systems with the microelectrode array in a multi-fountain pen array configuration could open new horizons for fast, simple, and high resolution write and read-out scanning of almost any interface. For instance, multi-fountain pen scanners could be used in forensic sciences for human fingerprint imaging at the crime scene.

Furthermore, more complicated microfluidic systems can be prepared by adding supplementary open microchannels for the aspiration of the flowing redox mediator solution to avoid sample contamination encountered during scanning with a fountain pen device. Moreover, such channels might also be useful to deliver minute solution volumes for further analyses to mass spectrometers, chromatographs, electrophoresis, or other analytical instruments. As a consequence, online monitoring of electrochemical and chemical surface information can be foreseen. SECM imaging in a contact regime is likely possible on curved samples as well as under weightlessness conditions by adjusting the attractive forces at the interfaces between redox mediator solution, probe, and sample surface.

Soft SECM probes can be fabricated with different approaches, such as photolithography, in which a wafer-level batch fabrication method is used to reproducibly produce standard soft stylus probes. To perform SECM experiments in organic solvents, polymeric materials chemically more stable than PET can be used for the microfabrication of soft probes. For instance, polyimide can be employed for the preparation of fountain pen 
probes for SECM experiments in dichloroethane or acetonitrile. Less demanding techniques might be used for the preparation of microelectrodes simply by printing processes.

In summary, the time has come for imaging chemical reactivity on substrates of different shapes and sizes and for SECM to become a routine imaging tool with applications ranging from biological tissue imaging to process control.

\section{BIOGRAPHY}

Hubert H. Girault is Director of the Laboratory of Physical \& Analytical Chemistry at the Ecole Polytechnique Fédérale de Lausanne, Switzerland. He is also Adjunct Professor at the Engineering Research Center of Innovative Scientific Instruments at Fudan University, Shanghai, China. His research interests include electrochemistry at liquid-liquid interfaces, Off-gel electrophoresis and protein fractionation, electrochemical aspects of ionization methods for mass spectrometry, and SECM. Gunther Wittstock is a full professor of physical chemistry at the C. v. Ossietzky University of Oldenburg. He and his group are using SECM and spectroelectrochemistry for the study of functional systems at electrode surfaces that range from sensing layers to catalytic surfaces for energy conversion. Fernando Cortés Salazar got recently his $\mathrm{PhD}$ at the laboratory of professor Girault at EPFL, where currently is working as a post-doc. His research interests are focused on the development of new probes for SECM, corrosion science, electrophoretic separations of proteins and lab on a chip applications. Dmitry Momotenko is currently a $\mathrm{PhD}$ student under the supervision of Prof. Girault at EPFL. His main research activities involve fabrication of new probes for scanning electrochemical microscope and numerical simulations of mass-transport phenomenon in electrolyte solutions. Andreas Lesch is a $\mathrm{PhD}$ student at the Carl v. Ossietzky University of Oldenburg where he is working on implementation and application of approaches to parallel SECM imaging. Contact Girault at hubert.girault@epfl.ch; +4121-693 3145 (tel.); +41-21-693 3667 (fax).

\section{ACKNOWLEDGMENT}

The Fonds National Suisse pour la Recherche Scientifique (Grant no 20PA21_121570/1) and Deutsche Forschungsgemeinschaft (Wi 1617/10) are thanked for financial support of the joint project "High throughput SECM imaging". The technical assistance by Valérie Devaud and Cyrille Hibert is also acknowledged.

\section{REFERENCES}

(1) Engstrom, R. C.; Weber, M.; Wunder, D. J.; Burgess, R.; Winquist, S. Anal. Chem. 1986, 58, 844-848.

(2) Liu, H. Y.; Fan, F. R. F.; Lin, C. W.; Bard, A. J. J. Am. Chem. Soc. 1986, 108, 3838-3839.

(3) Barker, A. L.; Gonsalves, M.; MacPherson, J. V.; Slevin, C. J.; Unwin, P. R. Anal. Chim. Acta 1999, 385, 223-240.

(4) Wittstock, G.; Hesse, R.; Schuhmann, W. Electroanalysis 1997, 9, 746-750.

(5) Yasukawa, T.; Kaya, T.; Matsue, T. Electroanalysis 2000, 12, 653659.

(6) Sun, P.; Mirkin, M. V. Anal. Chem. 2006, 78, 6526-6534.

(7) Fushimi, K.; Seo, M. Zairyo to Kankyo 1997, 46, 797-803.

(8) Walsh, D. A.; Fernandez, J. L.; Bard, A. J. J. Electrochem. Soc. 2006, 153, E99-E103.

(9) Zhang, M.; Girault, H. H. Analyst 2009, 134, 25-30.
(10) Mandler, D. In Scanning Electrochemical Microscopy; Bard, A. J., Mirkin, M. V., Eds.; Marcel Dekker: New York, Basel., 2001; pp 593-627.

(11) Kwak, J.; Bard, A. J. Anal. Chem. 1989, 61, 1221-1227.

(12) Slevin, C. J.; Macpherson, J. V.; Unwin, P. R. J. Phys. Chem. B 1997, 101, 10851-10859.

(13) Cornut, R.; Lefrou, C. J. Electroanal. Chem. 2008, 621, 178-184.

(14) Sun, P.; Laforge, F. O.; Mirkin, M. V. Phys. Chem. Chem. Phys. 2007, 9, 802-823.

(15) Wittstock, G.; Burchardt, M.; Pust, S. E.; Shen, Y.; Zhao, C. Angew. Chem., Int. Ed. 2007, 46, 1584-1617.

(16) Scott, E. R.; White, H. S.; Bradley Phipps, J. J. Membr. Sci. 1991, $58,71-87$.

(17) Fan, F. R. F.; Bard, A. J. Science 1995, 270, 1849-1852.

(18) Forouzan, F.; Bard, A. J. J. Phys. Chem. B 1997, 101, 1087610879 .

(19) Cortes-Salazar, F.; Traeuble, M.; Li, F.; Busnel, J.-M.; Gassner, A.; Hojeij, M.; Wittstock, G.; Girault, H. H. Anal. Chem. 2009, 81, 6889-6896.

(20) Cortés-Salazar, F.; Lesch, A.; Momotenko, D.; Busnel, J.-M.; Wittstock, G.; Girault, H. H. Anal. Methods 2010, 2, 817-823.

(21) Cortés-Salazar, F.; Momotenko, D.; Lesch, A.; Wittstock, G.; Girault, H. H. Anal. Chem. 2010, DOI: 10.1021/ac1019304.

(22) Wei, C.; Bard, A. J.; Nagy, G.; Toth, K. Anal. Chem. 1995, 67, 1346-1356.

(23) Wipf, D. O.; Bard, A. J. Anal. Chem. 1992, 64, 1362-1367.

(24) Wipf, D. O.; Bard, A. J.; Tallman, D. E. Anal. Chem. 1993, 65, 1373-1377.

(25) Macpherson, J. V.; Unwin, P. R. Anal. Chem. 2000, 72, 276-285.

(26) Kranz, C.; Friedbacher, G.; Mizaikofft, B.; Lugstein, A.; Smoliner, J.; Bertagnolli, E. Anal. Chem. 2001, 73, 2491-2500.

(27) Sklyar, O.; Kueng, A.; Kranz, C.; Mizaikoff, B.; Lugstein, A.; Bertagnolli, E.; Wittstock, G. Anal. Chem. 2005, 77, 764-771.

(28) Treutler, T. H.; Wittstock, G. Electrochim. Acta 2003, 48, 29232932.

(29) Kueng, A.; Kranz, C.; Lugstein, A.; Bertagnolli, E.; Mizaikoff, B. Angew. Chem., Int. Ed. 2003, 42, 3238-3240.

(30) Burt, D. P.; Wilson, N. R.; Weaver, J. M. R.; Dobson, P. S.; Macpherson, J. V. Nano Lett. 2005, 5, 639-643.

(31) Ludwig, M.; Kranz, C.; Schuhmann, W.; Gaub, H. E. Rev. Sci. Instrum. 1995, 66, 2857-2860.

(32) Ballesteros Katemann, B.; Schulte, A.; Schuhmann, W. Chem. Eur. J. 2003, 9, 2025-2033.

(33) Alpuche-Aviles, M. A.; Wipf, D. O. Anal. Chem. 2001, 73, 48734881.

(34) Ballesteros, K. B.; Schulte, A.; Calvo, E. J.; Koudelka-Hep, M.; Schuhmann, W. Electrochem. Commun. 2002, 4, 134-138.

(35) Diakowski, P. M.; Ding, Z. Electrochem. Commun. 2007, 9, 2617-2621.

(36) Lohrengel, M. M.; Moehring, A.; Pilaski, M. Fresenius J. Anal. Chem. 2000, 367, 334-339.

(37) Spaine, T. W.; Baur, J. E. Anal. Chem. 2001, 73, 930-938.

(38) Turcu, F.; Schulte, A.; Schuhmann, W. Anal. Bioanal. Chem. 2004, 380, 736-741.

(39) Bratten, C. D. T.; Cobbod, P. H.; Cooper, J. M. Anal. Chem. 1997, 69, 253-258.

(40) Juncker, D.; Schmid, H.; Delamarche, E. Nat. Mater. 2005, 4, 622-627.

(41) Eklund, S.; Hedlund, L. J. Sci. Instrum. 1951, 28, 30.

(42) Lewis, A.; Kheifetz, Y.; Shambrodt, E.; Radko, A.; Khatchatryan, E.; Sukenik, C. Appl. Phys. Lett. 1999, 75, 2689-2691.

(43) Deladi, S.; Tas, N. R.; Berenschot, J. W.; De Boer, J. H.; De Boer, M. J.; Krijnen, G. J. M.; Elwenspoek, M. C. Proceedings of the IEEE International Conference on Micro Electro Mechanical Systems (MEMS) 2005, 564-567.

(44) Shin, C.; Hwang, K.; Kim, Y.; Kim, H. In 20th International Microprocesses and Nanotechnology Conference 2007, 2007; pp 502-503.

(45) Leïchlé, T.; Nicu, L.; Descamps, E.; Corso, B.; Mailley, P.; Livache, T.; Bergaud, C. Appl. Phys. Lett. 2006, 88, 254108.

(46) Wipf, D. O.; Bard, A. J. J. Electrochem. Soc. 1991, 138, 469-474. 
(47) Nunes Kirchner, C.; Traeuble, M.; Wittstock, G. Anal. Chem. 2010, 82, 2626-2635.

(48) Zhang, M.; Wittstock, G.; Shao, Y.; Girault, H. H. Anal. Chem. 2007, 79, 4833-4839.

(49) Black, M.; Cooper, J.; McGinn, P. Meas. Sci. Technol. 2005, 16, 174-182.

(50) Fernandez, J. L.; Walsh, D. A.; Bard, A. J. J. Am. Chem. Soc. 2005, 127, 357-365.

(51) Bard, A. J. J. Am. Chem. Soc. 2010, 132, 7559-7567.

(52) Combellas, C.; Fermigier, M.; Fuchs, A.; Kanoufi, F. Anal. Chem. 2005, 77, 7966-7975.

(53) Liu, W.; Ye, H.; Bard, A. J. J. Phys. Chem. C 2010, 114, 12011207.

(54) Zoski, C. G.; Simjee, N.; Guenat, O.; Koudelka-Hep, M. Anal. Chem. 2004, 76, 62-72.

(55) Hong, S.; Mirkin, C. A. Science 2000, 288, 1808-1811.

(56) Vettiger, P.; Despont, M.; Drechsler, U.; Dorig, U.; Haberle, W.; Lutwyche, M. I.; Rothuizen, H. E.; Stutz, R.; Widmer, R.; Binnig, G. K. IBM J. Res. Dev. 2000, 44, 323-340.

(57) Meyer, H.; Drewer, H.; Gründig, B.; Cammann, K.; Kakerow, R.; Manoli, Y.; Mokwa, W.; Rospert, M. Anal. Chem. 1995, 67, 11641170.

(58) Zhang, L.; Ma, X. Z.; Zhuang, J. L.; Qiu, C. K.; Du, C. L.; Tang, J.; Tian, Z. W. Adv. Mater. 2007, 19, 3912-3918.

(59) Barker, A. L.; Unwin, P. R.; Gardner, J. W.; Rieley, H. Electrochem. Commun. 2004, 6, 91-97.

(60) Stulik, K.; Amatore, C.; Holub, K.; Marecek, V.; Kutner, W. Pure Appl. Chem. 2000, 72, 1483-1492.

(61) Bond, A. M.; Oldham, K. B.; Zoski, C. G. Anal. Chim. Acta 1989, $216,177-230$

(62) Wittstock, G.; Grondig, B.; Strehlitz, B.; Zimmer, K. Electroanalysis 1998, 10, 526-531.

(63) Cortés-Salazar, F.; Busnel, J.-M.; Li, F.; Girault, H. H. J. Electroanal. Chem. 2009, 635, 69-74.

(64) Zhang, M.; Girault, H. H. Electrochem. Commun. 2007, 9, 17781782.

(65) Cortes-Salazar, F.; Zhang, M.; Becue, A.; Busnel, J.-M.; Prudent, M.; Champood, C.; Girault, H. H. Chimia 2009, 63, 580. 